\title{
A FORTRAN Computer Program for Converting Among Atom, Weight, and Oxide Percentages for Use in Analytical Electron Microscopy
}

\author{
W.C. BIGELOW \\ Department of Materials and Metallurgical Engineering, \\ University of Michigan, Ann Arbor, Michigan 48109
}

\begin{abstract}
KEY WORDS Computer program chemical formula calculations, oxide percent calculations
\end{abstract}

\begin{abstract}
A FORTRAN program is given which will (1) calculate atom and weight percentages from a given chemical formula: (2) calculate atom percentages from given weight percentages; (3) calculate oxide percentages from given weight percentages; and (4) calculate weight percentages from given oxide percentages. The program is interactive, easy to use, and very convenient for use in electron beam microanalytical work.
\end{abstract}

\section{INTRODUCTION}

Many scanning electron microscopes are now equipped with either energy-dispersive or crystal spectrometer systems capable of carrying out quantitative chemical analyses. When working with such systems it is frequently desirable to make conversions among weight, atom, and oxide percentages. This is particularly true when dealing with ceramic, mineral, and intermetallic phases. Such conversions are tedious to perform by hand, and most computer-based analytic systems do not provide a program for carrying them out.

The program CCC (Chemical Composition Converter) listed here allows the following conversions to be carried out in a straightforward manner for phases containing up to 15 elements:
(1) conversion from chemical formula to atomic and weight percent,

(2) conversion from atom percent to weight percent,

(3) conversion from weight percent to oxide percent, and

(4) conversion from oxide percent to weight percent.

The program is written in FORTRAN, but is organized so that it should be easily trans. lated into BASIC. Each of the above conversion processes is handled in a separate subroutine so any not needed in a particular laboratory can be omitted. The subroutine for conversion from chemical formula to atomic and weight percentages will accept noninteger coefficients to facilitate calculations for phases involving chemical substitution. This is illustrated in the examples of the program's output shown below.

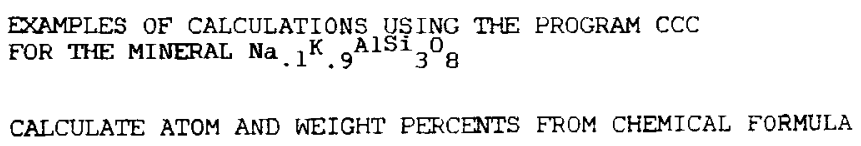

$\begin{array}{lcr}\text { ELMT } & \text { ATNO } & \text { ATMC WT } \\ \text { NA } & 11 & 22.990 \\ \text { K } & 19 & 39.102 \\ \text { AL } & 13 & 26.982 \\ \text { SI } & 14 & 28.086 \\ \text { O } & 8 & 15.999\end{array}$

$\begin{array}{rrrl}\text { \#/MOL } & \text { AT PCT } & \text { WT PCT } & \text { EL } \\ 0.10 & 0.769 & 0.831 & \text { NA } \\ 0.90 & 6.923 & 12.717 & \text { K } \\ 1.00 & 7.692 & 9.751 & \text { AL } \\ 3.00 & 23.077 & 30.449 & \mathrm{SI} \\ 8.00 & 61.538 & 46.253 & \mathrm{O}\end{array}$

Received January 10, 1985; accepted March 24, 1985.

Address reprint requests to W.C. Bigelow, Dept. of Materials and Metallurgical Engineering, Dow Building, 1500 Hayward Street, University of Michigan, Ann Arbor, MI 48109. 
CALCULATE OXIDE PERCENT FROM WEIGHT PERCENT

$\begin{array}{lrrrrrr}\text { ELMT } & \text { ATNO } & \text { ATOM WT } & \text { WT PCT } & \text { OXIDE } & \text { OX PCT } & \text { EL } \\ \text { NA } & 11 & 22.990 & 0.831 & \text { NA2O1 } & 1.120 & \text { NA } \\ \text { K } & 19 & 39.102 & 12.717 & \text { K 201 } & 15.319 & \text { K } \\ \text { AL } & 13 & 26.982 & 9.751 & \text { AL203 } & 18.424 & \text { AL } \\ \text { SI } & 14 & 28.086 & 30.449 & \text { SI102 } & 65.139 & \text { SI } \\ \text { WT PCT } & \text { OXYGEN }= & 46.253 & \text { TOTAL }= & 100.002 & \end{array}$

CALCULATE WEIGHT PERCENT FROM OXIDE PERCENT

$\begin{array}{ccrrrrr}\text { ELMT } & \text { ATNO } & \text { ATMC WT } & \text { OXIDE } & \text { OX PCT } & \text { WT PCT } & \text { EL } \\ \text { NA } & 11 & 22.990 & \text { NA2OI } & 1.120 & 0.831 & \text { NA } \\ \text { K } & 19 & 39.102 & \text { K } 201 & 15.319 & 12.717 & \text { K } \\ \text { AL } & 13 & 26.982 & \text { AL203 } & 18.424 & 9.751 & \text { AL } \\ \text { SI } & 14 & 28.086 & \text { SI1O2 } & 65.139 & 30.449 & \text { SI } \\ \text { WT PCT } & \text { OXYGEN }=46.254 & \text { TOTAL }=100.002 & \end{array}$

CALCULATE ATOM PERCENT FROM WEIGHT PERCENT

\begin{tabular}{|c|c|c|c|c|c|}
\hline ELMT & ATNO & ATMC W'T & WT PCT & AT PCT & EL \\
\hline NA & 11 & 22.990 & 0.831 & 0.769 & NA \\
\hline K & 19 & 39.102 & 12.717 & 6.923 & $\mathrm{~K}$ \\
\hline$A L$ & 13 & .982 & 9.751 & 7.693 & $A L$ \\
\hline SI & 14 & 28.086 & 30.449 & 23.077 & SI \\
\hline 0 & $\begin{array}{r}8 \\
\text { OTALS }\end{array}$ & 15 & $\begin{array}{r}46.25 \\
100.001\end{array}$ & $\begin{array}{r}61.538 \\
100.000\end{array}$ & 0 \\
\hline
\end{tabular}

CCC POURCE.CCC

C W. C. BIGELOW. UNIVERSITY OF MICHIGAN

C WRITE $(5,11)$

11 FORMAT $(2 \mathrm{X} /, 6 \mathrm{X}$, 'CCC, A PROG TO CONVERT AMONG WT.' (a' ATOM AND OXIDE PCTS')

10 WRITE $(5,1)$

1 FORMAT $(2 \mathrm{X} / 6 \mathrm{X}$,'DO YOU WANT TO CALCULATE:'/9X, (a' $1=W T$ \& ATM PCTS FROM CHEM FORMULA ' $/ 9 \mathrm{X}$.

@' $2=$ WT PCT FROM OXIDE PCT' $/ 9 X$.

a. 3=OXIDE PCT FROM WT PCT CATIONS'/9X.

a' 4=ATOM PCT FROM WT PCT / $/ 9 X$.

d. PKESS RETURN KEY TO STOP' $/ 2 \times / 6 \mathrm{X}$.

C

(a'TYPE NO. OF CHOICE'.\$)

$\operatorname{READ}(5.2) \mathrm{N}$

2 FORMAT(II)

GOTO $(70.20,30,40.50) \mathrm{N}+1$

GOTO 10

20 CALL WTCH

GOTO 10

30 CALL WTOX

GOTO 10

40 CALL OXWT GOTO 10

50 CALL ATWT

GOTO 10

70 CONTINUE

END 


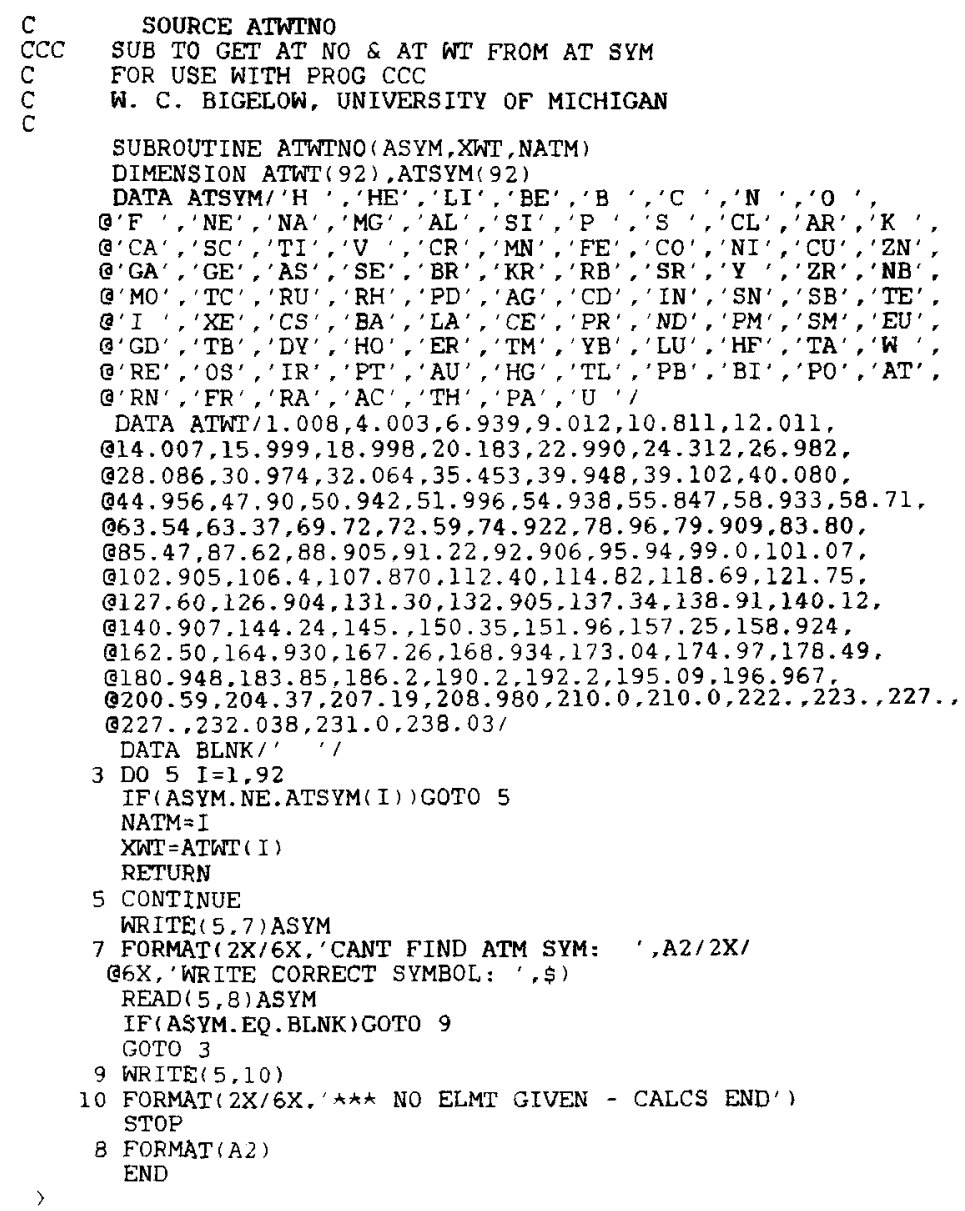

SOURCE. ATWT

SUB TO CONVERT FROM WT PCT TO ATM PCT

FOR USE WITH PROG CCC

W.C. BIGELOW. UNIVERSITY OF MICHIGAN

SUBROUTINE ATWT

COMMON /CBL/SYM(15), AWT(15),NZ (15), ELPCT(15)

DATA BLNK /

1 Do $15 \quad I=1,15$

WRITE $(5,3)$ I

3 FORMAT 2 X/6X.'SYMBOL FOR ELMT NO. ',I2.': ',\$)

READ ( 5, 4) SYM (I)

4 FORMAT(A2)

IF (SYM (I).NE. BLNK) GOTO 6

$\mathrm{JNUM}=\mathrm{I}-1$

GOTO 19

6 ASYM $=$ SYM( I)

CALL ATWTNO (ASYM, XWT, NATM)

$\operatorname{AWT}(I)=X W T$

$\mathrm{NZ}(I)=\mathrm{NATM}$ 
WRITE $(5,8)$

6 FORMAT (6X,'WT PCT THIS ELEMT',

READ ( 5,9 ) ELPCT ( I)

9 FORMAT (F8.0)

15 CONTINUE

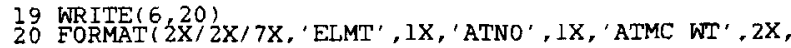
(d'WT PCT', $2 \mathrm{X}$, 'AT PCT', $2 \mathrm{X}$, 'EL')

C

SUM $=0.0$

21 DO $22 \mathrm{~J}=1$, JNUM

22 $\operatorname{SUM}=\operatorname{SUM}+(\operatorname{ELPCT}(\mathrm{J}) / \operatorname{AWT}(\mathrm{J}))$

WTOT $=0.0$

$\mathrm{ATOT}=0.0$

DO $28 \quad \mathrm{~J}=1 . \mathrm{JNUM}$

ATPCT $=(100.0 \star E L P C T(J) / A W T(J)) /$ SUM

ATOT $=$ ATOT + ATPCT

C

WTOT=WTOT+ELPCT $(\mathrm{J})$

WRITE $(6,25) S Y M(J), N Z(J), A W T(J), E L P C T(J), A T P C T, S Y M(J)$

25 FORMAT $(8 X, A 2,3 X, I 2,2 X, F 7.3,2 X, F 6,3,2 X, F 6.3,2 X, A 2)$

28 CONTINUE

WRITE $(6,31)$ WTOT, ATOT

31 FORMAT (IOX, 'TOTALS:',8X,F7.3,1X, [OF7.3/2X/2X/2X)

ENDFILE 6

RETURN

END

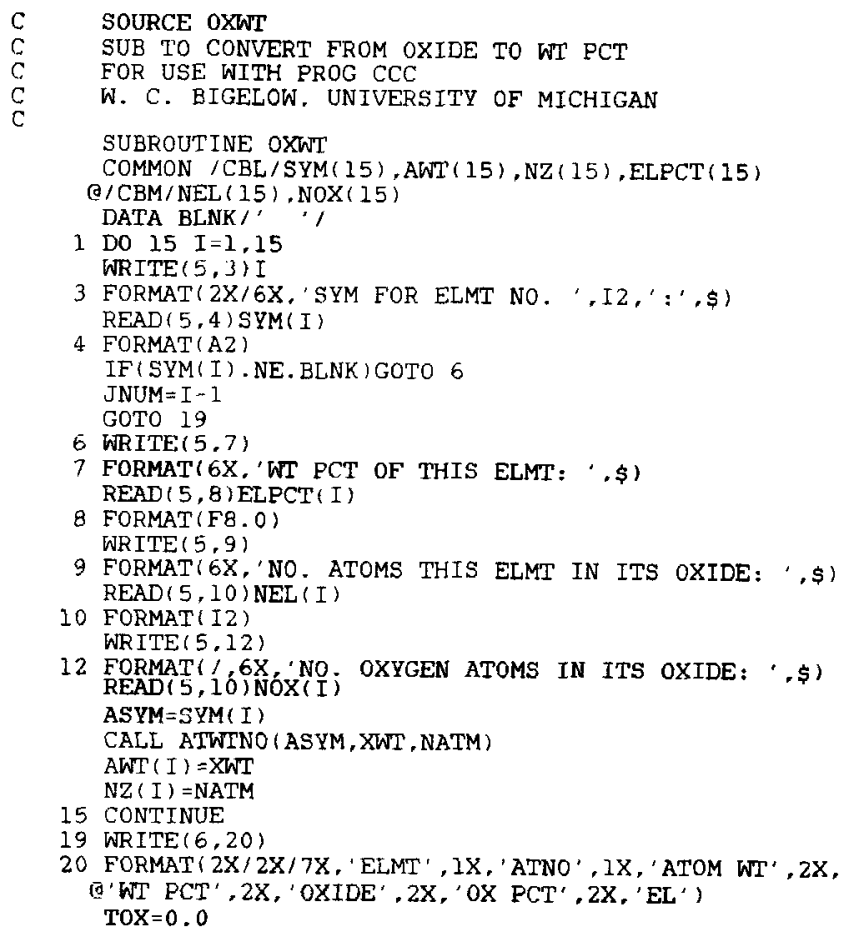




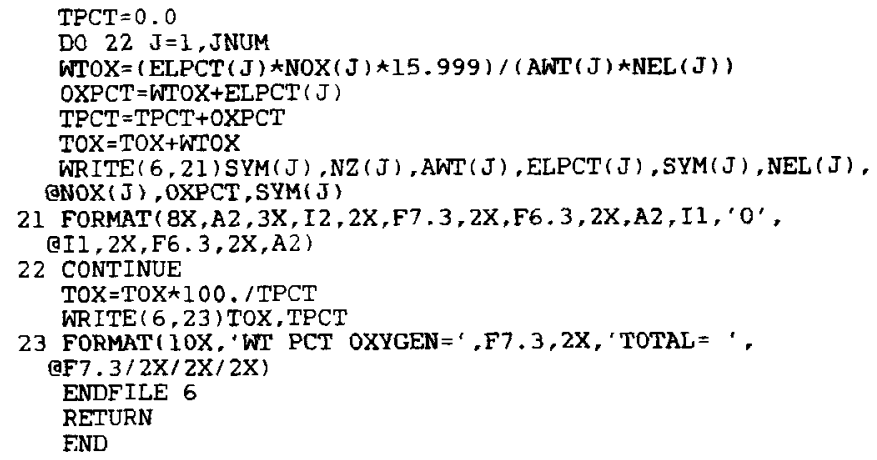




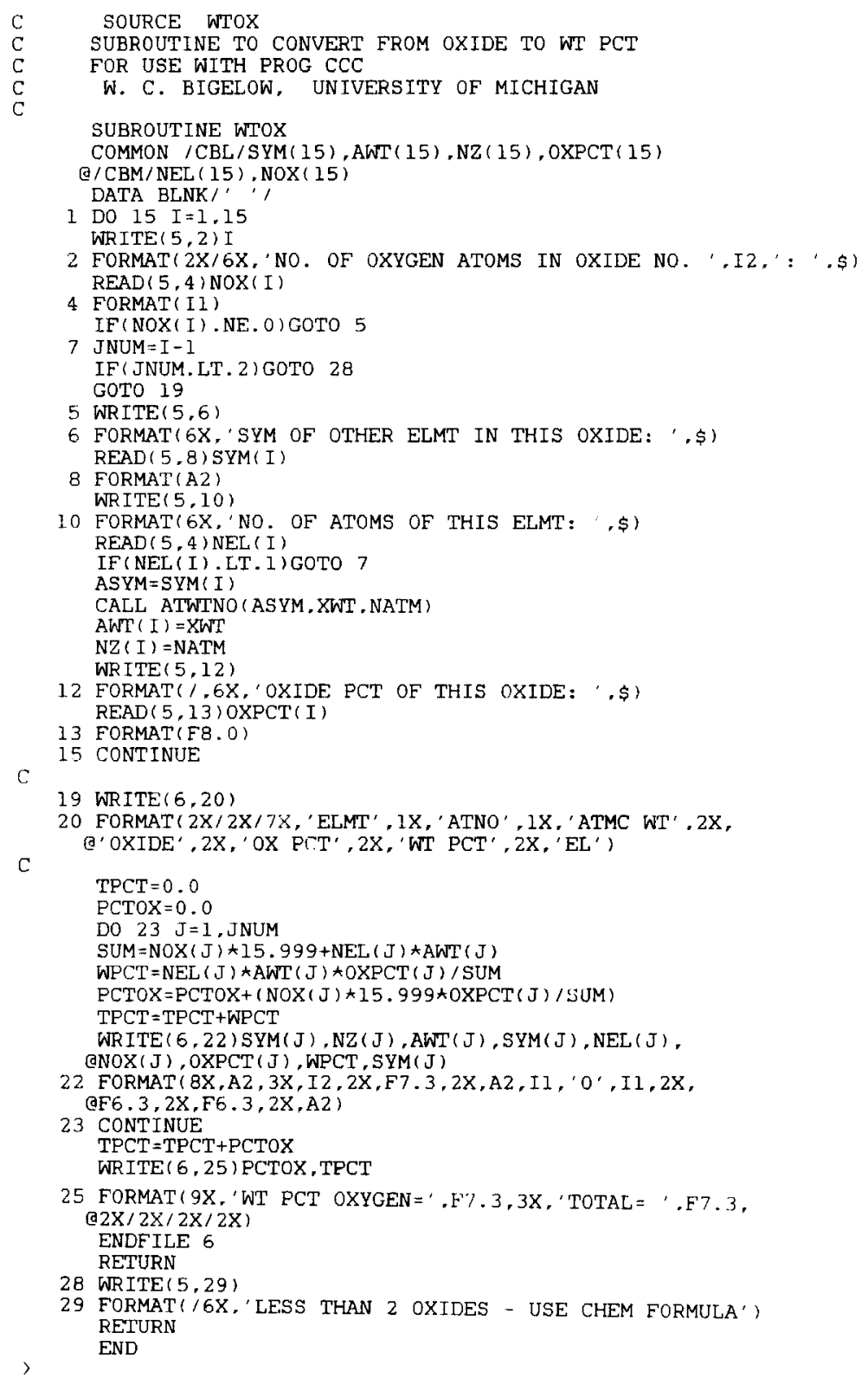

